\title{
Instability and spontaneous reconstruction of few-monolayer thick GaN graphitic structures (Supporting information)
}

\author{
A.V. Kolobov,$^{*, \dagger}$ P. Fons ${ }^{\dagger}$ J. Tominaga, ${ }^{\dagger}$ B. Hyot,,$^{\ddagger}$ and B. André $\neq$ \\ $\dagger$ Nanoelectronics Research Institute, National Institute of Advanced Industrial Science and \\ Technology (AIST), 1-1-1 Higashi, Tsukuba 305-8562, Japan \\ $\ddagger$ Université Grenoble Alpes, CEA, LETI, MINATEC campus, F38054 Grenoble, France. \\ E-mail: a.kolobov@aist.go.jp
}



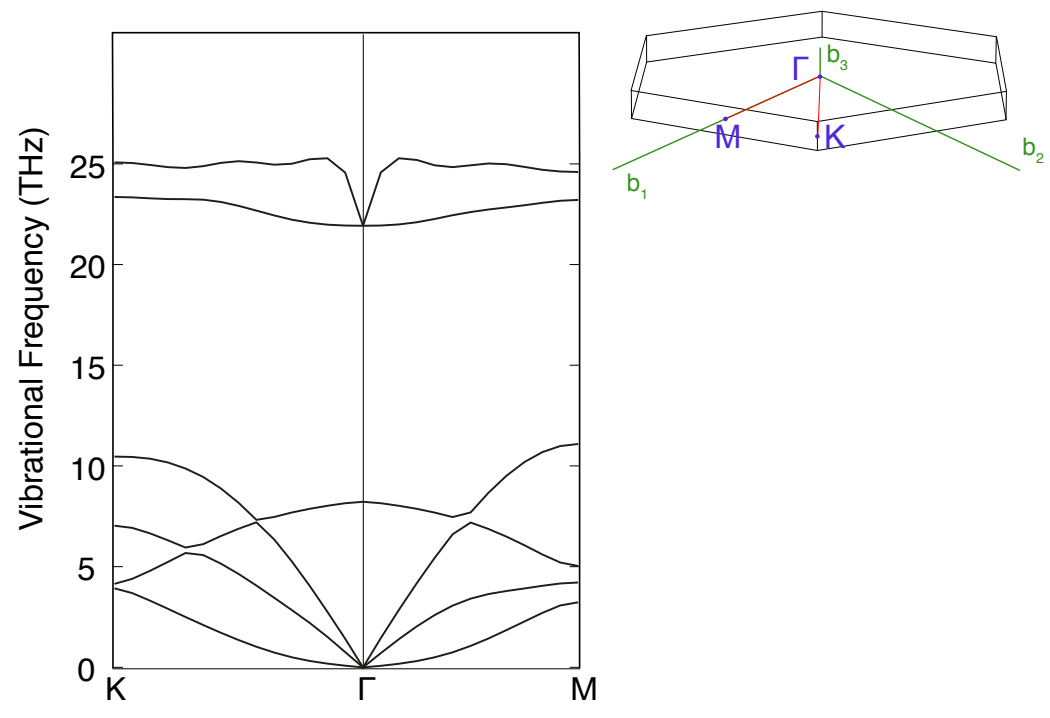

Figure S1: The phonon dispersion curve calculated for 1ML graphitic phase. The absence of imaginary modes demonstrates the structure stability 


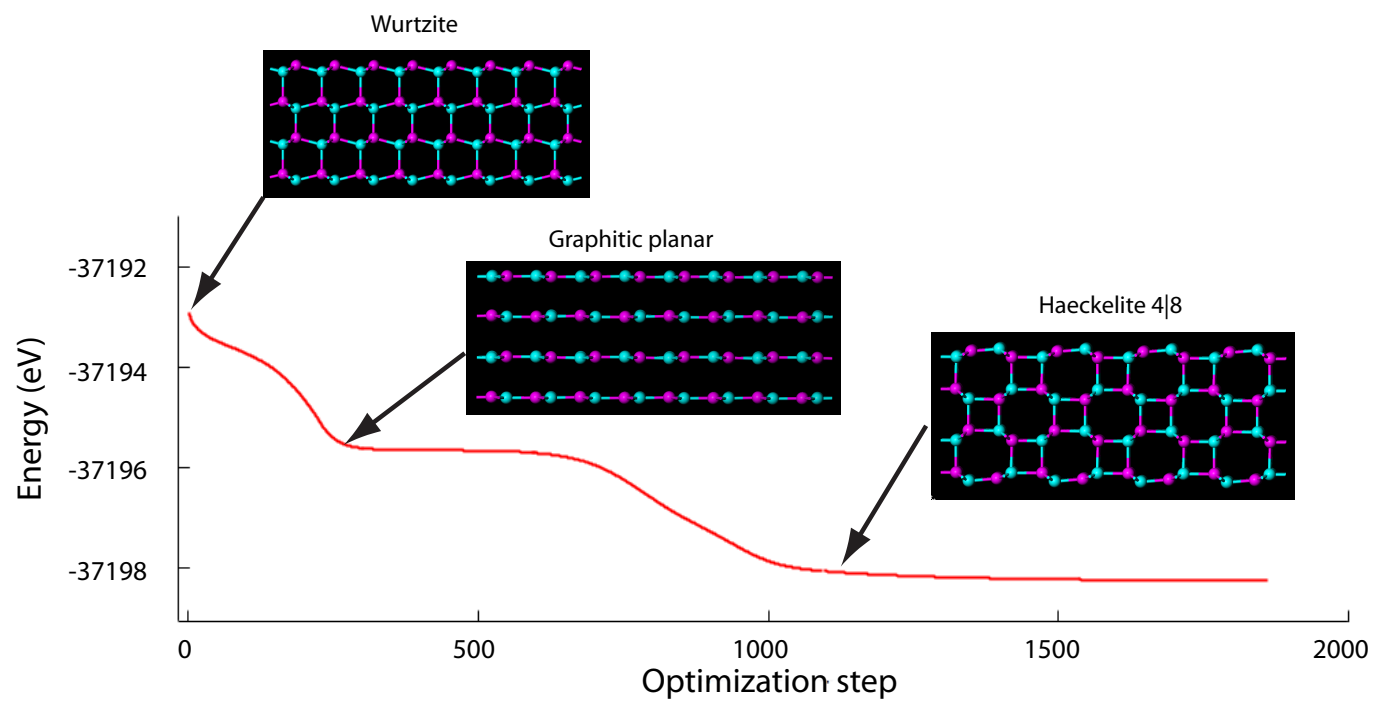

Figure S2: Structural evolution during the structure optimization at 0K. The starting $4 \mathrm{ML}$ wurtzite slab is unstable and readily changes into the graphitic structure. Since the latter is located at a saddle point in the energy landscape, the structure is trapped there between 300 and 1200 optimization steps, after which it eventually finds the lower energy Haeckelite phase. We note that the shown dependence does not have any physical meaning and is only used here to illustrate that a saddle-point structure may be apparently stable during the geometry optimization and can be mistaken for a local minimum. 